\title{
\Genome Biology
}

\section{Protein family review \\ The SR protein family}

\author{
Peter J Shepard and Klemens J Hertel
}

Address: Department of Microbiology and Molecular Genetics, University of California, Irvine, Irvine, CA 92697-4025, USA.

Correspondence: Klemens J Hertel. Email: khertel@uci.edu

\begin{abstract}
Summary
The processing of pre-mRNAs is a fundamental step required for the expression of most metazoan genes. Members of the family of serine/arginine (SR)-rich proteins are critical components of the machineries carrying out these essential processing events, highlighting their importance in maintaining efficient gene expression. SR proteins are characterized by their ability to interact simultaneously with RNA and other protein components via an RNA recognition motif (RRM) and through a domain rich in arginine and serine residues, the RS domain. Their functional roles in gene expression are surprisingly diverse, ranging from their classical involvement in constitutive and alternative pre-mRNA splicing to various post-splicing activities, including mRNA nuclear export, nonsense-mediated decay, and mRNA translation. These activities point up the importance of SR proteins during the regulation of mRNA metabolism.
\end{abstract}

\section{Gene organization and evolutionary history}

The discovery of SR proteins goes back to studies in Drosophila where genetic screens identified SWAP (suppressor-of-white-apricot) [1], Tra (transformer) [2] and Tra-2 (transformer-2) [3] as splicing factors. Their sequence characterization led to the identification of a protein domain rich in arginine and serine dipeptides, termed the arginine/serine (RS) domain. Subsequent identification of the splicing factors SF2/ASF and $\mathrm{SC}_{35}$ from human cell lines also revealed the presence of extended $\mathrm{RS}$ domains in addition to at least one RNAbinding domain of the RNA recognition motif (RRM)-type [4-6]. The family of SR proteins was classified following the identification of additional RS-domain-containing proteins on the basis of the presence of a phosphoepitope recognized by the monoclonal antibody mAb104 [7], their conservation across vertebrates and invertebrates, and their activity in splicing complementation assays [8]. In humans, the SR protein family is encoded by nine genes, designated splicing factor, arginine/serine-rich (SFRS) 1-7, 9, and 11 (Table 1). All nine members of the human SR protein family - SF2/ASF, SC35, SRp20, SRp40, SRp55, SRp75, SRp3oc, 9G8, and SRp54 - have a common structural organization (Figure 1), containing either one or two amino-terminal RNA-binding domains that provide RNA-binding specificity, and a variable-length RS domain at their carboxyl terminus that functions as a protein interaction domain [9].
More recent genome-wide studies have identified several other RS-domain-containing proteins, most of which are conserved in higher eukaryotes and function in pre-mRNA splicing or RNA metabolism [10]. Because of differences in domain structure, lack of mAb104 recognition, or lack of a prototypical RRM, these proteins are referred to as SR-like or SR-related proteins. An extensive list of SR-related proteins and their functional roles in RNA metabolism was recently discussed [11].

While introns are common to all eukaryotes, the complexity of alternative splicing varies among species. SR proteins exist in all metazoan species [8] as well as in some lower eukaryotes, such as the fission yeast Schizosaccharomyces pombe $[12,13]$. However, classical SR proteins are not present in all eukaryotes and are apparently missing from the budding yeast Saccharomyces cerevisiae, which lacks alternative splicing. Instead, three SR-like proteins have been identified in $S$. cerevisiae, one of which has been shown to modulate the efficiency of pre-mRNA splicing [14]. In general, the species-specific presence of SR proteins correlates with the presence of RS domains within other components of the general splicing machinery. The observation that the density of RS repeats correlates with the conservation of the branch-point signal, a critical sequence element of the 3' splice site, argues for an ancestral origin of SR proteins [15]. As such, SR proteins appear to be ancestral to eukaryotes and were subsequently lost independently in some lineages (Figure 2). Phylogenetic tree analyses further suggest that successive gene duplications played an important role in SR protein evolution [16]. These duplication events are coupled with high rates of nonsynonymous substitutions that promoted positive selection favoring the gain of new functions, supporting the hypothesis that the expansion of $\mathrm{RS}$ repeats during evolution had a fundamental role in the relaxation of the splicing signals and in the evolution of regulated splicing.

\section{Characteristic structural features}

All SR proteins share two main structural features: the RS domain and at least one RRM (Figure 1). For the majority of SR proteins with two RNA-binding domains, the second is a poor match to the RRM consensus and is referred to as an RRM homolog (RRMH). The only exception is 9G8, 
Table 1

\begin{tabular}{lccc}
\hline \multicolumn{4}{l}{ Human genes encoding SR proteins } \\
\hline Gene name & SR protein & Chromosomal location & UniProt \\
\hline SFRS1 & SF2/ASF/SRp30a & $17 q 21.3-q 22$ & Q07955 \\
SFRS2 & SC35/SRp30b & $17 q 25.1$ & Q01130 \\
SFRS3 & SRp20 & $6 p 21.31$ & P84103 \\
SFRS4 & SRp75 & $1 \mathrm{p} 35.3$ & Q08170 \\
SFRS5 & SRp40 & $14 q 24.2$ & Q13243 \\
SFRS6 & SRp55 & $20 q 13.11$ & Q13247 \\
SFRS7 & 9G8 & $2 \mathrm{p} 22.1$ & Q16629 \\
SFRS9 & SRp30c & $12 q 24.23$ & Q13242 \\
SFRS11 & SRp54 & $1 \mathrm{p} 31.1$ & Q05519
\end{tabular}

which contains an RRM and a zinc-knuckle domain that is thought to contact the RNA [17]. In the cases where it has been determined, SR proteins have specific, yet degenerate RNA-binding specificities [18,19]. The RS domains of SR proteins participate in protein interactions with a number of other RS-domain-containing splicing factors [20,21]. These include other SR proteins, SR-related proteins [22], and components of the general splicing machinery [20,21, 23-25]. Furthermore, the RS domain can function as a nuclear localization signal by mediating the interaction with the SR protein nuclear import receptor, transportin-SR [26-28].

Structural characterization of a complete SR protein has not yet been achieved. Consequently, only isolated RRMs of SR proteins have been analyzed structurally by nuclear magnetic resonance spectroscopy. Unfortunately, no structural information detailing the RS domain is available to date. This may be explained by the poor solubility of these proteins in their free state and the unknown phosphorylation state of the serines within the RS domain. In addition, the degenerate RNA-binding sequences recognized by SR proteins may have prevented their study in the bound form. To tackle the solubility issues, the RRMs of SRp2O and 9G8 were fused to the immunoglobulin G-binding domain 1 of Streptococcal protein G (GB1) solubility tag [29] or overexpressed RRMs were suspended in a solution containing charged amino acids [30]. Using these manipulations it was possible to obtain solution structures of the free 9G8 and SRp2O RRMs and of the SRp20 RRM in complex with the RNA sequence 5'CAUC-3' (Figure 3). When examining the unbound RRMs of SRp2O and 9G8, one is struck by an unusually large exposed hydrophobic surface, which could explain why the solubility of SR proteins is so low. The SRp20 RRM complex with RNA shows that although all four nucleotides present are contacted by the RRM, only the 5' cytosine is recognized in a specific manner. These

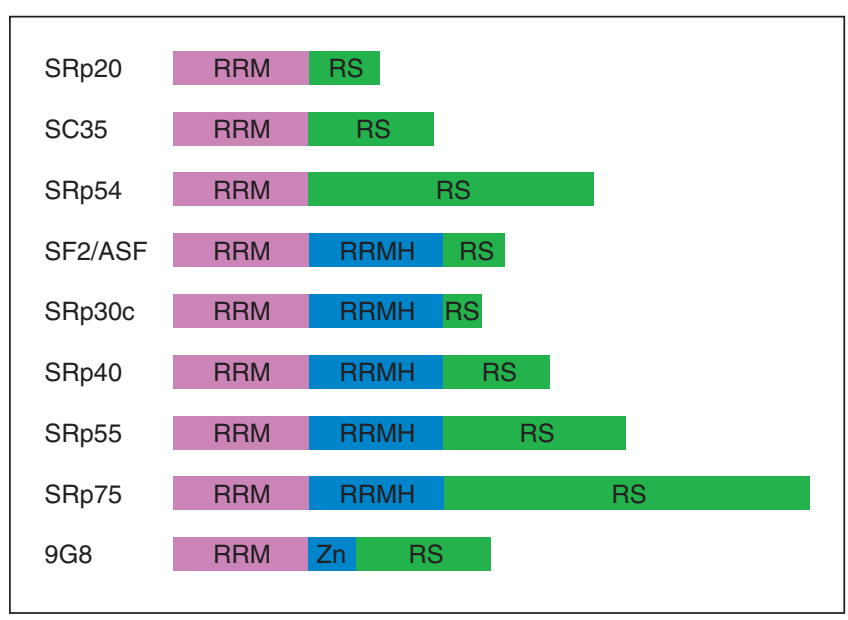

Figure 1

The human SR protein family. The structural organization of the nine human SR proteins is shown. RRM, RNA recognition motif; $\mathrm{RRMH}$, RRM homology; RS, arginine/serine-rich domain; Zn, Zinc knuckle.

structural insights provided an explanation for the seemingly low specificity of RNA binding exhibited by SRp2o [31,32].

\section{Localization and function}

Many proteins involved in pre-mRNA splicing, including the SR proteins, are enriched in nuclear compartments termed speckles, which occur throughout the nucleus. Speckles are of two distinct structural types [33]: interchromatin granule clusters (IGCs) about 20-25 nm in diameter, which are storage/reassembly sites for premRNA splicing factors; and perichromatin fibrils approximately $5 \mathrm{~nm}$ in diameter, which are sites of actively transcribing genes and co-transcriptional splicing [34]. The SR proteins are a prominent component of nuclear speckles (Figure 4) [35,36], and biochemical analyses have indicated that RS domains are responsible for targeting the SR proteins to these structures [26,37]. The intranuclear organization of SR proteins is dynamic, and they are recruited from the IGC storage clusters to the sites of co-transcriptional splicing, the perichromatin fibrils $[38,39]$. Interestingly, both the RNA-binding domains and RS domains are required for recruitment of SR proteins from the IGCs to the perichromatin fibrils, as is phosphorylation of the RS domain [40].

\section{Splicing activation}

In classic cases of alternative splicing, it has been shown that cis-acting RNA sequence elements, known as splicing enhancers, increase exon inclusion by serving as sites for recruitment of the splicing machinery - the spliceosome which is a complex of ribonucleoprotein splicing factors, such as U1 and U2 small nuclear ribonucleoproteins 
(a) Human SR proteins

$\overline{0.083}$

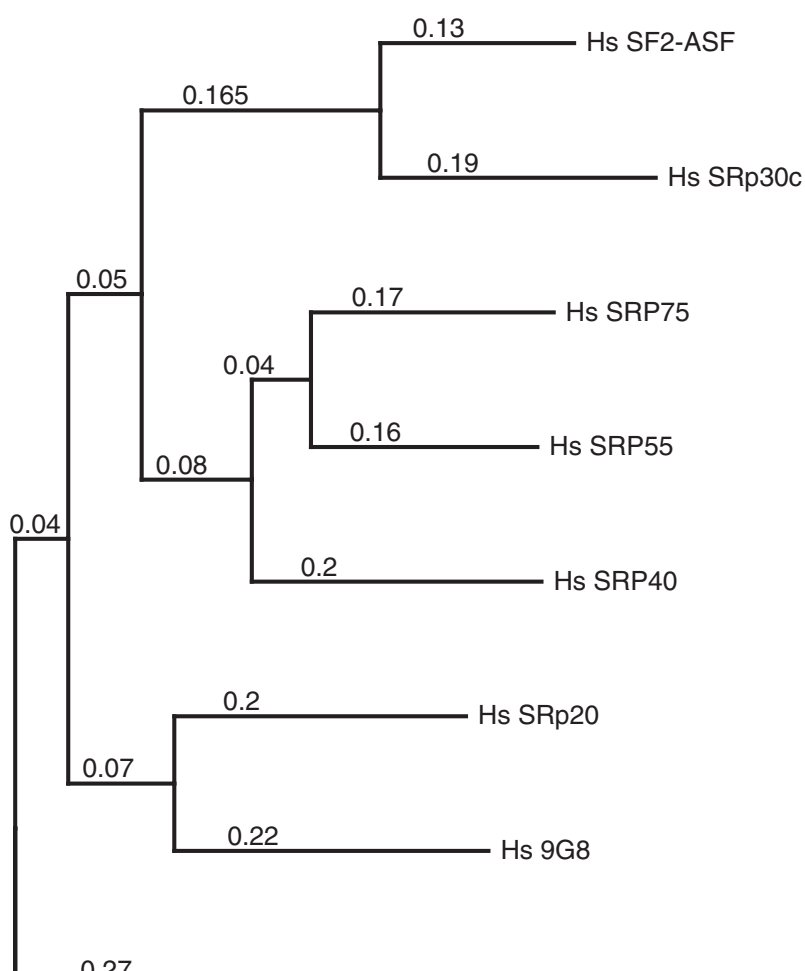

0.27

0.42 (b) Main species tree

$\overline{0.1335}$

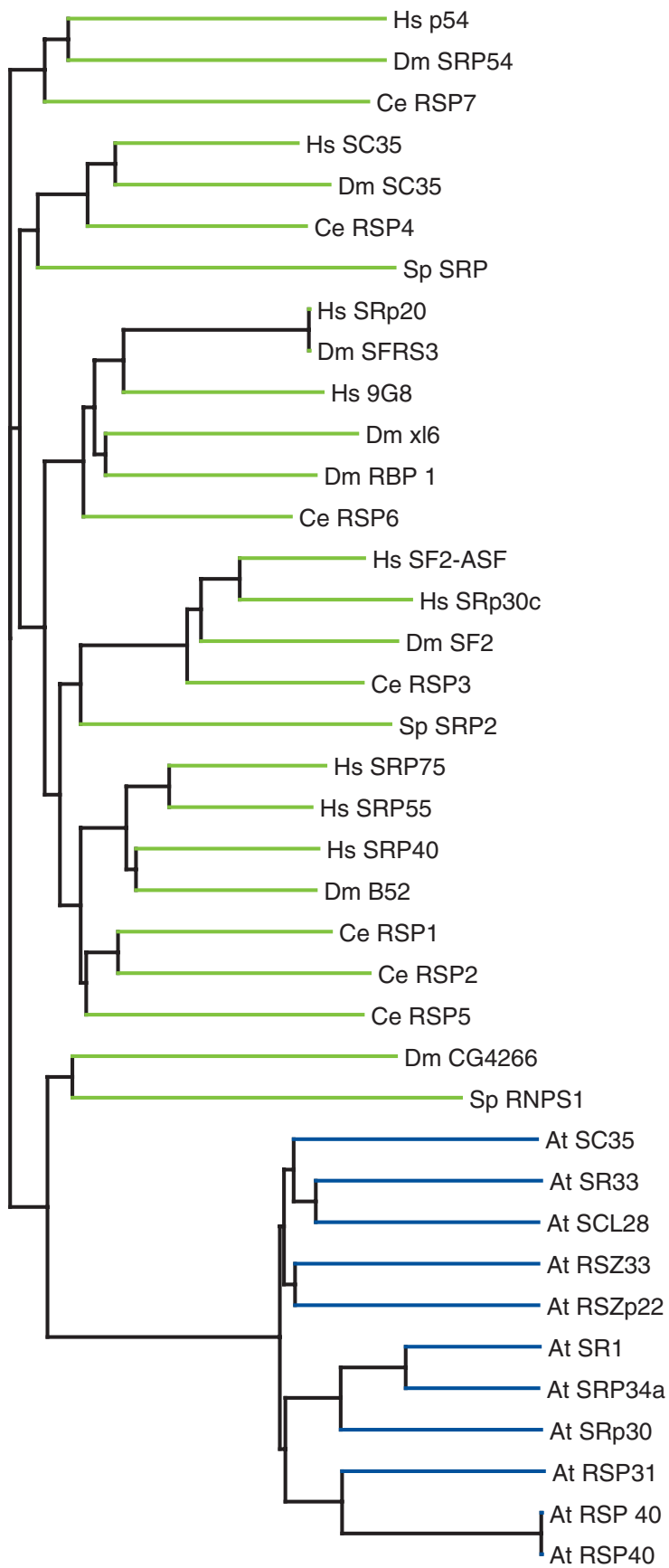

\section{Figure 2}

Evolutionary relationship between members of the SR family. The phylogeny was inferred using the neighbor-joining method. ClustalW was used to align sequences and perform phylogenetic analysis. Trees were drawn by CTree. The horizontal lines in each panel indicate the similarity between SR proteins. (a) Phylogenetic tree based on the alignment of the human (Hs) SR protein family. The numbers above each bar indicate the degree of similarity. (b) Phylogenetic tree based on the alignment of Homo sapiens (Hs), Drosophila melanogaster (Dm),

Caenorhabditis elegans (Ce), Arabidopsis thaliana (At), and Schizosaccharomyces pombe (Sp) SR protein sequences. Green and blue lines indicate different clusters. Cluster set selection is based on minimizing the subtype diversity ratio, a measure that groups related subclasses. 


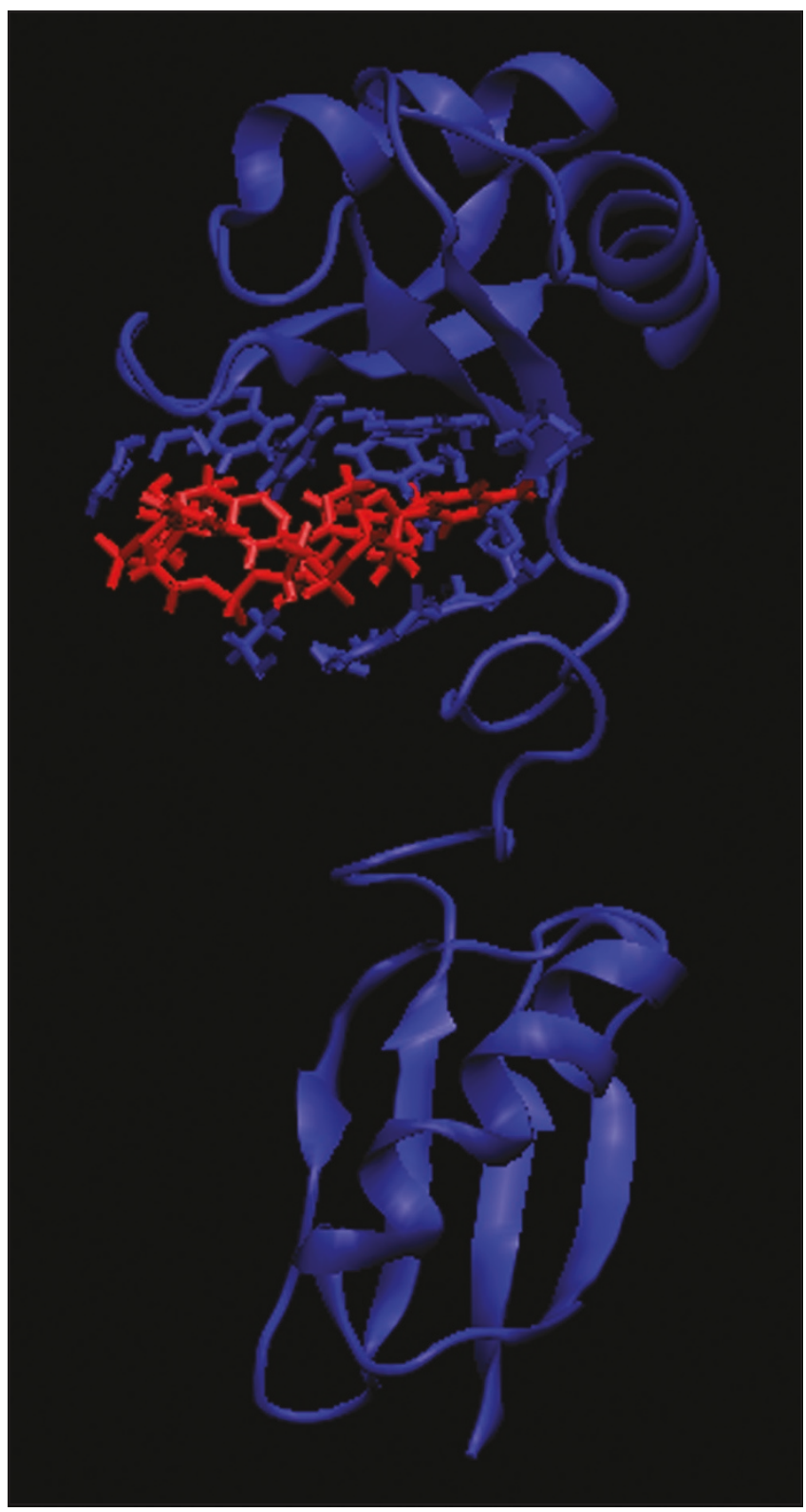

Figure 3

Solution structure of an SR protein RRM from human SRp20 (blue) in complex with the RNA sequence 5'-CAUC-3' (red). All four nucleotides present are contacted by the RRM, but only the 5' cytosine is recognized specifically. The structure was generated using the Visual Molecular Dynamics program [78] from coordinates deposited in the Brookhaven National Laboratory Protein Data Bank [30].

(snRNPs), and their associated proteins, such as U2 auxiliary factor (U2AF), that splices exons together and releases the intron RNA. Splicing enhancers are usually located within the regulated exon, and are thus known as exonic splicing enhancers (ESEs) [41,42]. ESEs are usually recognized by at least one member of the SR protein family and recruit the splicing machinery to the adjacent intron
[9,41,42]. SR proteins act at several steps during the splicing reaction $[4,5,8,43-45]$ and require phosphorylation for efficient splice-site recognition and dephosphorylation for splicing catalysis [46,47]. A number of SR protein kinases have been identified that specifically phosphorylate serine residues within the RS domain of SR proteins. These include SR protein kinase 1 (SRPK1) [48], Clk/Sty kinase [49], cdc2p34 [50], and topoisomerase [51]. Surprisingly, binding sites for SR proteins are not only limited to alternatively spliced exons, but have also been verified for exons of constitutively spliced pre-mRNAs [52,53]. It is therefore likely that SR proteins bind to sequences found in most, if not all, exons.

One model for the mechanism of splicing activation proposes that the RS domain of an enhancer-bound SR protein interacts directly with other splicing factors containing an RS domain, thus facilitating the recruitment of spliceosomal components such as the snRNP U1 to the 5' splice site or U2AF65 (the large subunit of the splicing factor U2AF) to the 3' splice site [9]. An alternative mode of spliceosomal recruitment was suggested by experiments showing that RS domains of SR proteins contact the premRNA within the functional spliceosome [54,55]. Irrespective of the RS domain activation mode, SR proteins facilitate the recruitment of spliceosomal components to the regulated splice site [42,56] (Figure 5a). Thus, SR proteins bound to ESEs function as general activators of exon definition [57]. Kinetic analyses have shown that the relative activity of ESE-bound SR proteins determines the magnitude of splicing promotion. This activity depended on the number of SR proteins assembled on an ESE and the distance between the ESE and the intron. It was also shown that activation of splicing was proportional to the number of serine-arginine repeats within the RS domain of the bound SR protein. Thus, the quantity of serine-arginine repeats appears to dictate the activation potential of SR proteins [58].

In addition to their exon-dependent functions, SR proteins have activities that do not require interaction with exon sequences [59]. The role of the exon-independent function may be to promote the pairing of 5' and 3' splice sites across the intron or to facilitate the incorporation of the tri-snRNP U4/U6•U5 into the spliceosome [44] (Figure 5b). $\mathrm{U}_{4} / \mathrm{U} 6 \cdot \mathrm{U}_{5}$ is a complex of snRNPs that contains the splicing activity. Although the RRM of the SR protein is essential for its exon-independent activity [59], it is likely that SR proteins interact with the partially assembled spliceosome or the tri-snRNP through RS domain contacts.

\section{Splicing repression}

One striking feature of SR proteins is their prevalent location within the pre-mRNA. In nearly all cases SR proteins have been found to interact with exonic sequences of the pre-mRNA. This is a surprising finding considering 

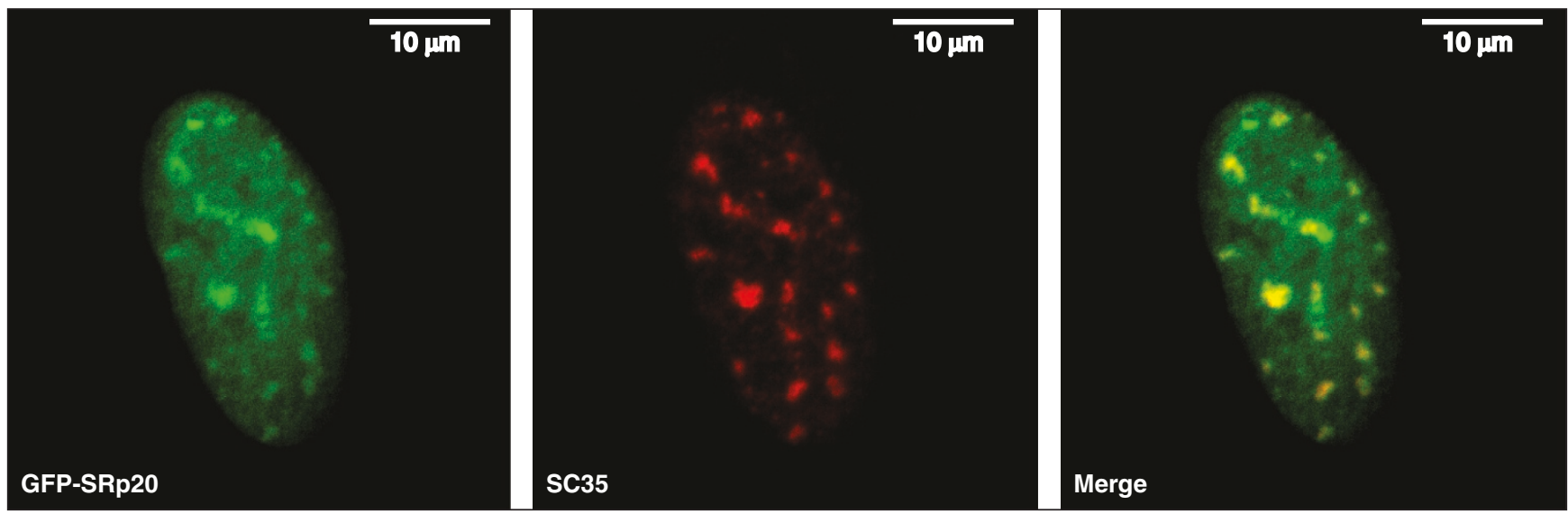

Figure 4

Localization of SR proteins within the nucleus. Left panel: HeLa cells transfected with GFP-SRp20. The GFP fluorescence is visualized directly. Middle panel: cells are also stained with anti-SC35 hybridoma supernatant to highlight clusters of SR proteins in the nucleus (red), which are referred to as nuclear speckles. Speckles are believed to be storage compartments for SR proteins and other splicing factors. Right panel: merge of GFP-SRp20 and SC35 images. The bar in each panel indicates the scale. Images courtesy of Lin Li and Rozanne Sandri-Goldin.

the fact that their relatively promiscuous binding specificity predicts that introns are littered with potential SR-protein-binding sites. The fact that SR proteins are mainly observed to bind within exonic sequences suggests that additional requirements need to be met for functional SR protein binding to the pre-mRNA. There are, however, some instances of SR proteins binding within the intron, where they function as negative regulators of splicing. The best-characterized example occurs during adenovirus infection [60]. In this case, splicing is repressed by the binding of the SR protein SF2/ASF to an intronic repressor element located upstream of the 3' splice site branchpoint sequence in the adenovirus pre-mRNA. When bound to the repressor element, $\mathrm{SF}$ /ASF prevents the recruitment of the snRNP U2 to the branchpoint sequence, thereby inactivating the 3' splice site (Figure 5c). Other studies have provided further support for the idea that SR proteins bound to introns generally interfere with the productive assembly of spliceosomes [61]. These observations show that exonic splicing enhancers not only function in exon and splice-site recognition, but also act as barriers to prevent exon skipping.

\section{Role of SR proteins in mRNA export}

Some SR proteins - SF2/ASF, SRp20, and 9G8 - shuttle continuously between the nucleus and the cytoplasm [62]. The movement of these proteins requires the phosphorylation of specific residues in the RS domain and the RNAbinding domain. These unique intracellular transport properties suggest that a subset of SR proteins functions not only in pre-mRNA processing but also in mRNA export [62]. In fact, the SR proteins 9G8 and SRp20 promote nuclear export of the intronless histone H2A mRNA in mammalian cells and Xenopus oocytes [63] by binding to a 22-nucleotide sequence within the H2A mRNA (Figure 6a). In addition, the $S$. cerevisiae protein Npl3p, which is closely related to the SR proteins, assists in mRNA export in yeast [64]. Once again, phosphorylation of specific serine residues within the RS domain seems to control the efficiency of the mRNA-export function of Npl3p [65]. Given the fact that SR proteins are essential for splicing [9], remain associated with the spliced mRNA after intron removal [66,67], and shuttle between the nucleus and the cytoplasm [62], it seems highly likely that SR proteins also play an important part in the export of spliced mRNAs. As shown recently, 9G8 and SRp20 are involved in mediating the efficient handover of mRNA to Tip-associated protein (TAP), which is an essential nuclear export factor [68].

\section{SR protein involvement in translation}

SR proteins have been shown to influence translation either indirectly or directly. For example, the splicing activity of SF2/ASF influences alternative splicing of the pre-mRNA for the protein kinase $\mathrm{MNK} 2$, a kinase that regulates translation initiation. High levels of SF2/ASF promote the production of an MNK2 mRNA isoform that enhances cap-dependent translation, whereas low levels achieve the opposite [69]. SF2/ASF is also involved in regulating translation directly. It has been shown to associate with polyribosome fractions isolated from cytoplasmic extracts and to enhance the translation efficiency of an ESE-containing luciferase reporter [70], apparently through mediating the recruitment of components of mTOR (mammalian target of rapamycin) signaling pathway (Figure 6b). As a result of this recruitment, a competitive inhibitor of cap-dependent translation is released [71]. 
(a)

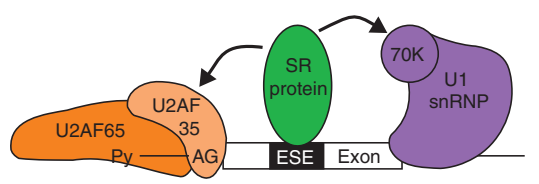

(b)

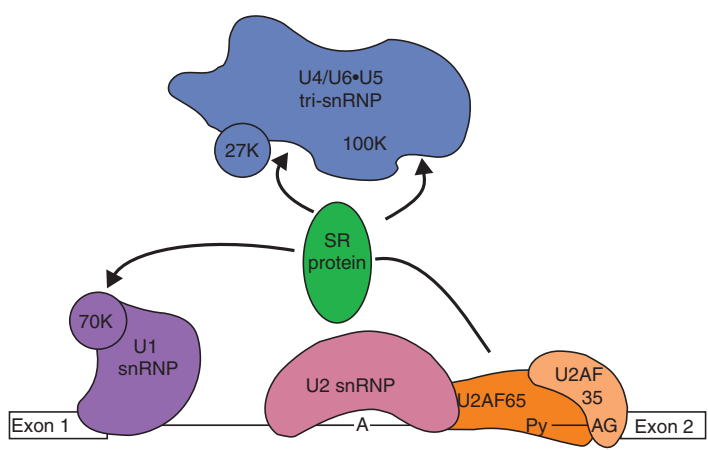

(c)

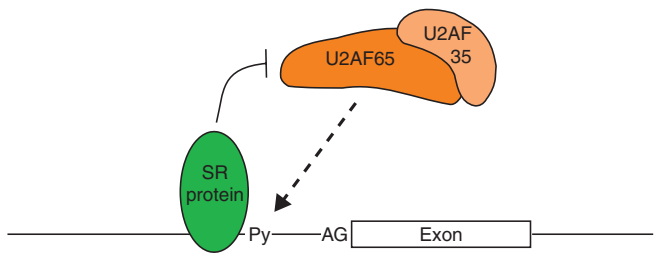

\section{Figure 5}

Splicing functions of SR proteins. (a) SR proteins (green) bound to an exonic splicing enhancer (ESE) may function in constitutive splicing by interacting with the splicing factors U2AF bound at the upstream 3' splice site and U1 snRNP bound to the downstream 5 ' splice site. Py represents the polypyrimidine tract, the binding site for U2AF. (b) Exon-independent functions of SR proteins. SR proteins may have two exon-independent functions. SR proteins facilitate splice-site pairing by simultaneously interacting with U1 snRNP and U2AF across the intron. SR proteins also assist in recruiting the U4/U6.U5 tri-snRNP. (c) Splicing repression is mediated when SR proteins associate with intronic sequences close to the splice sites. Recruitment of spliceosomal components is inhibited through steric hindrance or nonproductive spliceosomal assembly. Adapted with permission from [79].

Importantly, other SR proteins have also been reported to function in translation. SRp20 promotes translation of a viral RNA initiated at an internal ribosome entry site [72], and 9G8 increases translation efficiency of unspliced mRNA containing a constitutive transport element [73].

\section{Frontiers}

The functional characterization of SR proteins has revealed a wealth of information, placing SR proteins in the context of regulating constitutive and alternative pre-mRNA splicing, mediating efficient transport of mRNAs, and modulating mRNA translation. As such, SR proteins could easily be mistaken for 'Jacks of all trades, masters of none' in mRNA metabolism. However, many studies have demonstrated their essential presence in the cell, even with (a)

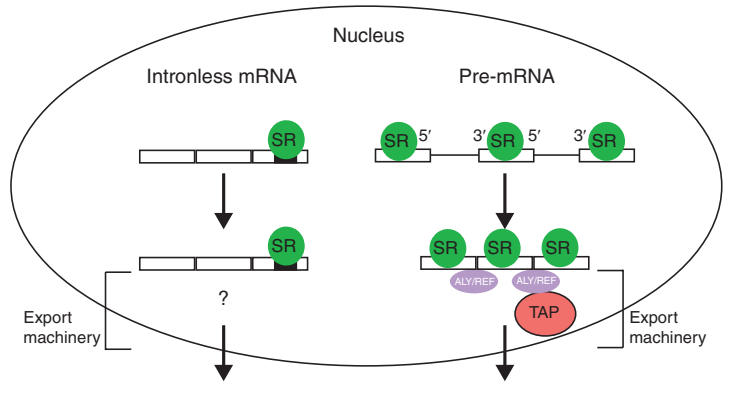

(b)

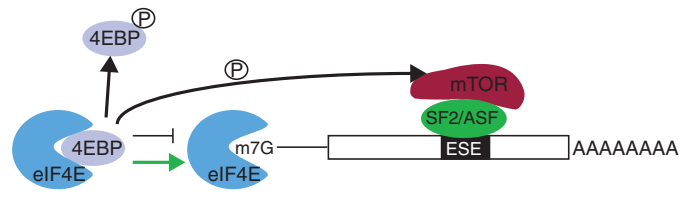

\section{Figure 6}

SR protein functions other than splicing. (a) mRNA export. SR proteins associate site-specifically with intronless mRNAs, such as histone H2A mRNA [63], to promote their export (left-hand side). The export machinery is as yet unknown. For intron-containing premRNAs (right-hand side), SR protein association with the spliced mRNA has also been suggested to mediate nuclear export through interactions with the RNA export factor ALY/REF and Tip-containing protein (TAP). (b) Translation initiation. Interactions between mRNA-bound SF2/ASF and the protein kinase mTOR trigger phosphorylation of 4E-BP (elF4E-binding protein). In its phosphorylated form 4E-BP dissociates from the translation initiation factor elF4E, thereby releasing elF4E and activating initiation of cap-dependent translation (green arrow) [71].

occasional redundancies. Given the enormous functional real estate this family of proteins covers, one is now pressed to find out how it is possible to transition these proteins between their involvements in the various steps of mRNA processing. Clearly, reversible modification, such as serine phosphorylation within the RS domain, is likely to be the ticket for SR protein functional flexibility [51]. The challenge will be to determine the extent and dynamics of such modifications within SR proteins specifically involved in one of these activities and whether changes in modification lend support to the existence of an SR protein-modification code, perhaps similar in principle to the now well-described histone-modification code [74].

An old foe makes up another challenge: SR protein structure. For more than 15 years attempts have been made to obtain high-resolution structures of SR proteins. So far, these attempts have failed because of problems of low solubility and the likely heterogeneity of RS-domain modifications. As a first step towards gaining ground in this endeavor, clever modification approaches have been used to obtain a high-resolution structure of the SR protein RRM domain. This is a significant first step. However, the 
much more elusive RS domain is still the big prize, requiring further creative approaches and manipulations to freeze this seemingly unstructured domain in a conformation that permits its structural elucidation.

A different and experimentally challenging puzzle to address is the balance between the relatively low RNAbinding specificity exhibited by SR proteins and their usually specific functional impact. Given that SR proteins generally associate with exon sequences, it is likely that their interaction with the RNA is often aided by other factors. This suggestion is supported by the observation that at least $75 \%$ of the nucleotides in a typical human exon are part of sequence motifs that have been found to influence splicing, presumably through the binding of splicing activators, such as SR proteins, or the binding of splicing repressors, such as heterogeneous nuclear RNPs [75]. For example, it is possible that the binding of SR proteins to pre-mRNA is only guaranteed if they are flanked by spliceosomal components such as U2 snRNP auxiliary factor or U1 snRNP, thus establishing a network of protein-protein and protein-RNA interactions. The establishment of such a network would then permit the stable association of SR proteins with many different target sequences, thus enabling SR proteins to recognize the thousands of different exons present in higher eukaryotes [76]. Therefore, the relatively low RNA-binding specificity may have evolved to uphold the suitability of SR proteins to participate effectively in multiple RNA-processing events.

Clearly, SR proteins make up a family of regulators with important functions in RNA metabolism. This realization is exemplified when considering that changes in SR protein function or abundance have frequently been associated with human disease. For example, SF2/ASF has been described as a proto-oncogene [69] and the misregulation of alternative splicing has been associated with several types of cancer [77]. While the involvement of SR proteins in various aspects of gene expression has been shown to be widespread, it would not be surprising if they emerge as critical players in other important biological processes.

\section{Acknowledgements}

We are grateful to the Hertel laboratory for helpful comments on the manuscript and Lin $\mathrm{Li}$ and Rozanne Sandri-Goldin for providing images of SR protein speckles. Our research is supported by $\mathrm{NIH}$ grant GM $62287(\mathrm{KJH})$.

\section{References}

1. Chou TB, Zachar Z, Bingham PM: Developmental expression of a regulatory gene is programmed at the level of splicing. EMBO J 1987, 6:4095-4104.

2. Boggs RT, Gregor P, Idriss S, Belote JM, McKeown M: Regulation of sexual differentiation in $D$. melanogaster via alternative splicing of RNA from the transformer gene. Cell 1987, 50:739-747.

3. Amrein $\mathrm{H}$, Gorman M, Nothiger R: The sex-determining gene tra-2 of Drosophila encodes a putative RNA binding protein. Cell 1988, 55:1025-1035.
4. Ge H, Manley JL: A protein factor, ASF, controls cell-specific alternative splicing of SV40 early pre-mRNA in vitro. Cell 1990, 62:25-34.

5. Krainer AR, Conway GC, Kozak D: Purification and characterization of pre-mRNA splicing factor SF2 from HeLa cells. Genes Dev 1990, 4:1158-1171.

6. Fu XD, Maniatis T: The 35-kDa mammalian splicing factor SC35 mediates specific interactions between U1 and U2 small nuclear ribonucleoprotein particles at the 3' splice site. Proc Natl Acad Sci USA 1992, 89:1725-1729.

7. Roth MB, Zahler AM, Stolk JA: A conserved family of nuclear phosphoproteins localized to sites of polymerase II transcription. J Cell Biol 1991, 115:587-596.

8. Zahler AM, Lane WS, Stolk JA, Roth MB: SR proteins: a conserved family of pre-mRNA splicing factors. Genes Dev 1992, 6:837-847.

9. Graveley BR: Sorting out the complexity of SR protein functions. RNA 2000, 6:1197-1211.

10. Boucher L, Ouzounis CA, Enright AJ, Blencowe BJ: A genomewide survey of RS domain proteins. RNA 2001, 7:16931701.

11. Long JC, Caceres JF: The SR protein family of splicing factors: master regulators of gene expression. Biochem $\mathrm{J}$ 2009, 417:15-27.

12. Lutzelberger M, Gross T, Kaufer NF: Srp2, an SR protein family member of fission yeast: in vivo characterization of its modular domains. Nucleic Acids Res 1999, 27:2618-2626.

13. Gross T, Richert K, Mierke C, Lutzelberger M, Kaufer NF: Identification and characterization of srp1, a gene of fission yeast encoding a RNA binding domain and a RS domain typical of SR splicing factors. Nucleic Acids Res 1998, 26:505-511.

14. Kress TL, Krogan NJ, Guthrie C: A single SR-like protein Npl3, promotes pre-mRNA splicing in budding yeast. Mol Cell 2008, 32:727-734.

15. Plass M, Agirre E, Reyes D, Camara F, Eyras E: Co-evolution of the branch site and SR proteins in eukaryotes. Trends Genet 2008, 24:590-594.

16. Escobar AJ, Arenas AF, Gomez-Marin JE: Molecular evolution of serine/arginine splicing factors family (SR) by positive selection. In Silico Biol 2006, 6:347-350.

17. Cavaloc Y, Popielarz M, Fuchs JP, Gattoni R, Stevenin J: Characterization and cloning of the human splicing factor 9G8: a novel $35 \mathrm{kDa}$ factor of the serine/arginine protein family. EMBO J 1994, 13:2639-2649.

18. Liu HX, Zhang M, Krainer AR: Identification of functional exonic splicing enhancer motifs recognized by individual SR proteins. Genes Dev 1998, 12:1998-2012.

19. Liu HX, Chew SL, Cartegni L, Zhang MQ, Krainer AR: Exonic splicing enhancer motif recognized by human SC 35 under splicing conditions. Mol Cell Biol 2000, 20:1063-1071.

20. Wu JY, Maniatis T: Specific interactions between proteins implicated in splice site selection and regulated alternative splicing. Cell 1993, 75:1061-1070.

21. Kohtz JD, Jamison SF, Will CL, Zuo P, Lührmann R, GarciaBlanco MA, Manley JL: Protein-protein interactions and 5' splice site recognition in mammalian mRNA precursors. Nature 1994, 368:119-124.

22. Blencowe BJ, Bowman JA, McCracken S, Rosonina E: SR-related proteins and the processing of messenger RNA precursors. Biochem Cell Biol 1999, 77:277-291.

23. Teigelkamp S, Mundt C, Achsel T, Will CL, Luhrmann R: The human U5 snRNP-specific 100-kD protein is an RS domaincontaining, putative RNA helicase with significant homology to the yeast splicing factor Prp28p. RNA 1997, 3: 1313-1326.

24. Fetzer S, Lauber J, Will CL, Luhrmann R: The [U4/U6.U5] trisnRNP-specific $27 \mathrm{~K}$ protein is a novel SR protein that can be phosphorylated by the snRNP-associated protein kinase. RNA 1997, 3:344-355.

25. Makarova OV, Makarov EM, Luhrmann R: The 65 and $110 \mathrm{kDa}$ SR-related proteins of the U4/U6.U5 tri-snRNP are essen- 
tial for the assembly of mature spliceosomes. EMBO $J$ 2001, 20:2553-2563.

26. Cáceres JF, Misteli T, Screaton GR, Spector DL, Krainer AR: Role of the modular domains of SR proteins in subnuclear localization and alternative splicing specificity. J Cell Biol 1997, 138:225-238.

27. Kataoka N, Bachorik JL, Dreyfuss G: Transportin-SR, a nuclear import receptor for SR proteins. J Cell Biol 1999, 145:11451152.

28. Lai MC, Lin RI, Huang SY, Tsai CW, Tarn WY: A human importin-beta family protein, transportin-SR2, interacts with the phosphorylated RS domain of SR proteins. $J$ Biol Chem 2000, 275:7950-7957.

29. Zhou P, Lugovskoy AA, Wagner G: A solubility-enhancement tag (SET) for NMR studies of poorly behaving proteins. $J$ Biomol NMR 2001, 20:11-14.

30. Hargous Y, Hautbergue GM, Tintaru AM, Skrisovska L, Golovanov AP, Stevenin J, Lian LY, Wilson SA, Allain FH: Molecular basis of RNA recognition and TAP binding by the SR proteins SRp20 and 9G8. EMBO J 2006, 25:5126-5137.

31. Cavaloc $\mathrm{Y}$, Bourgeois CF, Kister L, Stevenin J: The splicing factors 9 G8 and SRp20 transactivate splicing through different and specific enhancers. RNA 1999, 5:468-483.

32. Schaal TD, Maniatis T: Selection and characterization of pre-mRNA splicing enhancers: identification of novel SR protein-specific enhancer sequences. Mol Cell Biol 1999, 19:1705-1719.

33. Spector DL: Macromolecular domains within the cell nucleus. Annu Rev Cell Biol 1993, 9:265-315.

34. Spector DL: Nuclear organization and gene expression. Exp Cell Res 1996, 229:189-197.

35. Manley JL, Tacke R: SR proteins and splicing control. Genes Dev 1996, 10:1569-1579.

36. Fu X-D: The superfamily of arginine/serine-rich splicing factors. RNA 1995, 1:663-680.

37. Hedley ML, Amrein $\mathrm{H}$, Maniatis $\mathrm{T}$ : An amino acid sequence motif sufficient for subnuclear localization of an arginine/ serine-rich splicing factor. Proc Natl Acad Sci USA 1995, 92: 11524-11528.

38. Jimenez-Garcia LF, Spector DL: In vivo evidence that transcription and splicing are coordinated by a recruiting mechanism. Cell 1993, 73:47-59.

39. Misteli T, Caceres JF, Spector DL: The dynamics of a premRNA splicing factor in living cells. Nature 1997, 387:523527.

40. Misteli T, Caceres JF, Clement JQ, Krainer AR, Wilkinson MF, Spector DL: Serine phosphorylation of SR proteins is required for their recruitment to sites of transcription in vivo. J Cell Biol 1998, 143:297-307.

41. Blencowe BJ: Exonic splicing enhancers: mechanism of action, diversity and role in human genetic diseases. Trends Biochem Sci 2000, 25:106-110.

42. Black DL: Mechanisms of alternative pre-messenger RNA splicing. Annu Rev Biochem 2003, 72:291-336.

43. Fu X-D: Specific commitment of different pre-mRNAs to splicing by single SR proteins. Nature 1993, 365:82-85.

44. Roscigno RF, Garcia-Blanco MA: SR proteins escort the U4/ U6.U5 tri-snRNP to the spliceosome. RNA 1995, 1:692-706.

45. Chew SL, Liu HX, Mayeda A, Krainer AR: Evidence for the function of an exonic splicing enhancer after the first catalytic step of pre-mRNA splicing. Proc Natl Acad Sci USA 1999, 96:10655-10660.

46. Mermoud JE, Cohen P, Lamond Al: Ser/Thr-specific protein phosphatases are required for both catalytic steps of premRNA splicing. Nucleic Acids Res 1992, 20:5263-5269.

47. Mermoud JE, Cohen PT, Lamond Al: Regulation of mammalian spliceosome assembly by a protein phosphorylation mechanism. EMBO J 1994, 13:5679-5688.

48. Mattaj IW: RNA processing. Splicing in space. Nature 1994, 372:727-728.
49. Colwill K, Pawson T, Andrews B, Prasad J, Manley JL, Bell JC Duncan PI: The Clk/Sty protein kinase phosphorylates SR splicing factors and regulates their intranuclear distribution. EMBO J 1996, 15:265-275.

50. Okamoto $Y$, Onogi $H$, Honda $R$, Yasuda $H$, Wakabayashi $T$, Nimura Y, Hagiwara M: cdc2 kinase-mediated phosphorylation of splicing factor SF2/ASF. Biochem Biophys Res Commun 1998, 249:872-878.

51. Soret J, Tazi J: Phosphorylation-dependent control of the pre-mRNA splicing machinery. Prog Mol Subcell Biol 2003, 31:89-126.

52. Schaal TD, Maniatis T: Multiple distinct splicing enhancers in the protein-coding sequences of a constitutively spliced pre-mRNA. Mol Cell Biol 1999, 19:261-273.

53. Fairbrother WG, Yeh RF, Sharp PA, Burge CB: Predictive identification of exonic splicing enhancers in human genes. Science 2002, 297:1007-1013.

54. Shen H, Kan JL, Green MR: Arginine-serine-rich domains bound at splicing enhancers contact the branchpoint to promote prespliceosome assembly. Mol Cell 2004, 13:367376.

55. Shen H, Green MR: A pathway of sequential arginine-serine-rich domain-splicing signal interactions during mammalian spliceosome assembly. Mol Cell 2004, 16:363-373.

56. Hertel KJ, Lynch KW, Maniatis T: Common themes in the function of transcription and splicing enhancers. Curr Opin Cell Biol 1997, 9:350-357.

57. Lam BJ, Hertel $\mathrm{KJ}$ : A general role for splicing enhancers in exon definition. RNA 2002, 8:1233-1241.

58. Graveley $B R$, Hertel $\mathrm{KJ}$, Maniatis $\mathrm{T}$ : A systematic analysis of the factors that determine the strength of pre- mRNA splicing enhancers. EMBO $J$ 1998, 17:6747-6756.

59. Hertel KJ, Maniatis $\mathrm{T}$ : Serine-arginine (SR)-rich splicing factors have an exon-independent function in pre-mRNA splicing. Proc Natl Acad Sci USA 1999, 96:2651-2655.

60. Kanopka A, Muhlemann O, Akusjarvi G: Inhibition by SR proteins of splicing of a regulated adenovirus pre- mRNA Nature 1996, 381:535-538.

61. Ibrahim EC, Schaal TD, Hertel KJ, Reed R, Maniatis T: Serine/ arginine-rich protein-dependent suppression of exon skipping by exonic splicing enhancers. Proc Natl Acad Sci USA 2005, 102:5002-5007.

62. Cáceres JF, Screaton GR, Krainer AR: A specific subset of Sr proteins shuttles continuously between the nucleus and the cytoplasm. Genes Dev 1998, 12:55-66.

63. Huang Y, Steitz JA: Splicing factors SRp20 and 9G8 promote the nucleocytoplasmic export of mRNA. Mol Cell 2001, 7: 899-905.

64. Lee MS, Henry M, Silver PA: A protein that shuttles between the nucleus and the cytoplasm is an important mediator of RNA export. Genes Dev 1996, 10:1233-1246.

65. Gilbert W, Siebel CW, Guthrie C: Phosphorylation by Sky1p promotes $\mathrm{Npl3p}$ shuttling and mRNA dissociation. RNA 2001, 7:302-313

66. Blencowe BJ, Nickerson JA, Issner R, Penman S, Sharp PA Association of nuclear matrix antigens with exon-containing splicing complexes. J Cell Biol 1994, 127:593-607.

67. Le Hir H, Moore MJ, Maquat LE: Pre-mRNA splicing alters mRNP composition: evidence for stable association of proteins at exon-exon junctions. Genes Dev 2000, 14:10981108.

68. Hautbergue GM, Hung ML, Golovanov AP, Lian LY, Wilson SA: Mutually exclusive interactions drive handover of mRNA from export adaptors to TAP. Proc Natl Acad Sci USA 2008 105:5154-5159.

69. Karni R, de Stanchina E, Lowe SW, Sinha R, Mu D, Krainer AR: The gene encoding the splicing factor SF2/ASF is a proto-oncogene. Nat Struct Mol Biol 2007, 14:185-193.

70. Sanford JR, Gray NK, Beckmann K, Caceres JF: A novel role for shuttling SR proteins in mRNA translation. Genes Dev 2004, 18:755-768. 
71. Michlewski G, Sanford JR, Caceres JF: The splicing factor SF2/ASF regulates translation initiation by enhancing phosphorylation of 4E-BP1. Mol Cell 2008, 3:179-189.

72. Bedard KM, Daijogo S, Semler BL: A nucleo-cytoplasmic SR protein functions in viral IRES-mediated translation initiation. EMBO J 2007, 26:459-467.

73. Swartz JE, Bor YC, Misawa Y, Rekosh D, Hammarskjold ML: The shuttling SR protein 9G8 plays a role in translation of unspliced mRNA containing a constitutive transport element. J Biol Chem 2007, 282:19844-19853.

74. Jenuwein T, Allis CD: Translating the histone code. Science 2001, 293:1074-1080.

75. Chasin LA: Searching for splicing motifs. Adv Exp Med Biol 2007, 623:85-106.
76. Hertel KJ: Combinatorial control of exon recognition. J Biol Chem 2008, 283:1211-1215.

77. Faustino NA, Cooper TA: Pre-mRNA splicing and human disease. Genes Dev 2003, 17:419-437.

78. Humphrey W, Dalke A, Schulten K: VMD: visual molecular dynamics. J Mol Graph 1996, 14:27-28, 33-38.

79. Graveley BR, Hertel KJ: SR proteins. In Encyclopedia of Life Sciences. Chichester: John Wiley \& Sons; 2005. doi:10.1038/ npg.els.0005039.

Published: 27 October 2009

doi:10.1186/gb-2009-10-10-242

(c) 2009 BioMed Central Ltd 